\title{
Influence of polarization on contact angle saturation during electrowetting
}

\author{
Chen Jiang, ${ }^{1}$ Hanbin Ma, ${ }^{1}$ David G. Hasko, ${ }^{1}$ and Arokia Nathan ${ }^{1, a)}$ \\ ${ }^{1}$ Department of Engineering, University of Cambridge, Cambridge, CB3 OFA, United Kingdom
}

Electrowetting is widely used to manipulate liquids on a dielectric surface by changing the wettability of the solid-liquid interface using an externally applied electric field. While the contact angle can be adequately predicted at low fields using Lippmann's model, there is a large disagreement with experimental behavior at high fields, where the contact angle saturates. Previous attempts to explain this saturation effect (by considering a range of different mechanisms) have led to models that are applicable only to limited field ranges. Here, we use a model for the solid-liquid interfacial surface energy (based on a dipole-dipole interaction) to describe electrowetting and find that this explains the contact angle change at both low (continuous change) and high (saturation) fields. The model is compared with measured contact angle changes for both water and ethylene glycol liquids, with good agreement over the whole field range.

a) Author to whom correspondence should be addressed. Electronic mail: an299@cam.ac.uk 
Electrowetting allows the wettability of an electrically conducting liquid on a dielectric surface to be manipulated by an externally applied electric field using a potential difference between the liquid and a counter electrode on the other face of the dielectric substrate. This technology is used in various applications such as optics, displays, and lab-on-a-chip systems ${ }^{1-5}$. An important limitation to this technology is that the change in contact angle with potential difference saturates at high applied voltages. Previous attempts to explain this effect have considered charge trapping in the dielectric layer ${ }^{6}$, non-negative solidliquid interfacial surface energy $y^{7,8}$, high electric field strengths at the contact line ${ }^{9}$, and generalized free energy ${ }^{10}$. However, none of these mechanisms explain the contact angle over both the low (continuous change) and high (saturation) voltage ranges. In this letter, we investigate the effect of dipole polarization (due to the applied potential difference) on interfacial surface energy and contact angle. The classical model to describe contact angle change with electric field is derived from Lippmann's equation, which gives the surface energy for a metal-electrolyte interface, based on electrostatic considerations $^{11,12}$. However, in situations where significant dipole-dipole polarization is possible, such an electrostatic approach may be inapplicable.

A typical electrowetting geometry is shown in Fig. 1(a). In the experiment described here this consists of a glass substrate with a 100-nm thick sputtered nickel layer to act as a grounding electrode; a 100-nm thick layer of silicon nitride $\left(\mathrm{SiN}_{\mathrm{x}}\right)$ deposited by PECVD (MVSystems LLC) at a substrate temperature of $150{ }^{\circ} \mathrm{C}$; and a thin hydrophobic dielectric layer, formed by spin-coating CYTOP (CTL-809M), diluted with a mass ratio of 1:3 to CYTOP solvent (CT-Solv. 180). Two test liquids were investigated, water and ethylene glycol. In both cases potassium chloride ( $\mathrm{KCl})$ was added to give a concentration of $0.1 \mathrm{mM}$ to increase conductivity, so that the conducting liquid could be contacted using a platinum electrode.

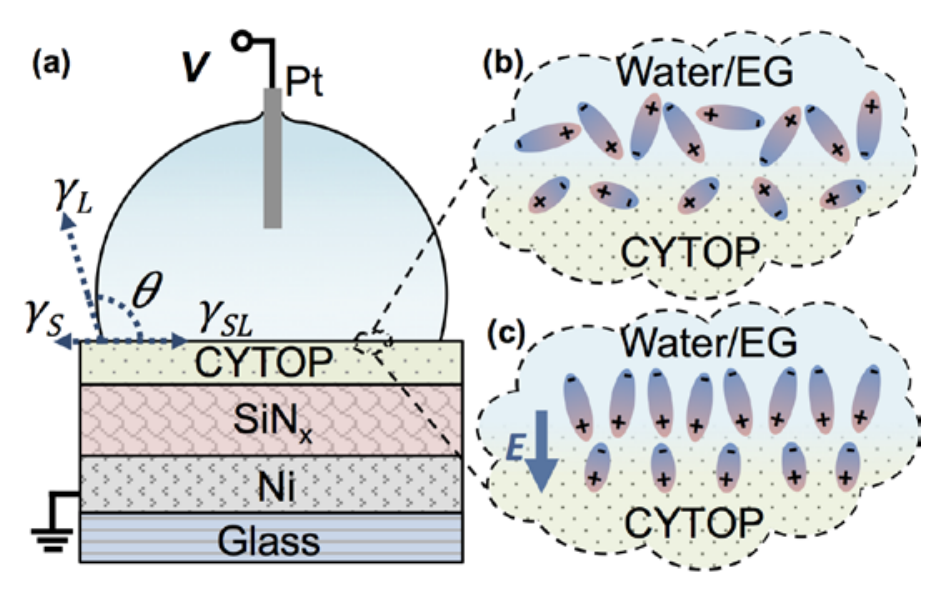

FIG. 1. (a) Schematic diagram of the electrowetting geometry. The relative orientation of dipoles at the solid-liquid interface are shown in (b) for the point of zero charge and in (c) under the influence of a positive applied voltage $V$. 
The contact angle $\theta$ is established by $\gamma_{L} \cos \theta=\gamma_{S}-\gamma_{S L}$ for mechanical equilibrium of the surface energies $\gamma_{S}$, $\gamma_{L}$, and $\gamma_{S L}$ at the intersection of the three phases (solid, liquid, and vapor) ${ }^{13}$. According to Fowkes' theory ${ }^{14}$, the surface energy can be written as a sum of components due to a number of nearly independent intermolecular interactions, including dispersion, polar, induction, hydrogen bonding, and metallic interactions. In the Lifshitz-van der Waals/Lewis acid-base (LW-AB) approach, these surface energy components are characterized by separate polar and apolar components. The apolar component is determined by the Good-Girifalco-Fowkes geometric mean combining rule ${ }^{14,15}$, while the polar component is determined by electron acceptor (Lewis acid) and electron donor (Lewis base) parameters ${ }^{16 .}$

$$
\begin{aligned}
\gamma_{S L} & =\left(\sqrt{\gamma_{S}^{L W}}-\sqrt{\gamma_{L}^{L W}}\right)^{2} \\
& +2\left(\sqrt{\gamma_{S}^{+}}-\sqrt{\gamma_{L}^{+}}\right)\left(\sqrt{\gamma_{S}^{-}}-\sqrt{\gamma_{L}^{-}}\right)
\end{aligned}
$$

where $i$ is for solid (S) or liquid (L), $\gamma_{i}^{L W}$ is the LW surface energy component, $\gamma_{i}^{+}$and $\gamma_{i}^{-}$are the acid and the base surface energy components at the point of zero charge (PZC), respectively.

Since the polar surface energy component originates from the interaction between Lewis acids and Lewis bases, it is greatly affected by the polarization of dipoles. As shown schematically in Figs. 1(b) and (c), changing the applied voltage from zero to a positive value increases the influence of the Lewis bases in the solid and increases the influence of the Lewis acids in the liquid at the solid-liquid interface. This modifies the interfacial surface energy due to the increase in dipole orientation close to the interface. But if the applied voltage is sufficiently large, no further dipole reorientation is possible and the interfacial surface energy will not change for further voltage increases, so that the contact angle change saturates.

The distribution of dipole orientations is assumed to follow Boltzmann statistics. An electric field causes the dipoles to rotate, so increasing alignment, but the thermal random motion tends to decrease alignment. For any given electric field $E$, the electrostatic energy of a dipole is determined by the rotational angle of the dipole with respect to the field, i.e. $U(\delta)=$ $-\mu E \cos \delta$, where $\mu$ is the dipole moment, and $\delta$ is the rotational angle of a dipole with respect to an applied electric field $E$, as shown in Fig. 2 inset. According to Boltzmann statistics, the density distribution of dipoles decreases with dipole electrostatic energy, i.e. $n(U(\delta))=A \exp \left(-\frac{U(\delta)}{k_{B} T}\right)=A \exp \left(\frac{\mu E \cos \delta}{k_{B} T}\right)$, where $A$ is normalizing constant, $k_{B}$ is Boltzmann constant, $T$ is absolute temperature. Therefore, the density function of dipole orientation can be written as $n(\delta$; $E)=$ $A \exp \left(\frac{\mu E \cos \delta}{k_{B} T}\right)$. The positive sign inside the exponent of this density function is resulted from the negative signs in both 
expressions of the Boltzmann distribution and the dipole electrostatic energy. For dipoles, the orientation can be characterized by the normalized mean of the scalar projection of dipole moment in the direction of the electric field, i.e.

$$
\alpha(E)=\frac{\int_{0}^{2 \pi} \int_{\pi}^{0} \mu \cos \delta n(\delta ; E) \sin \delta d \delta d \phi}{\mu}=L\left(\frac{\mu E}{k_{B} T}\right)
$$

where $L(x)$ is the Langevin function. The double integral is used due to three-dimensional rotation of dipoles, as shown in Fig. 2 inset. Once the dipoles are aligned, due to an induction interaction, the solid Lewis acids (bases) will bind to the liquid Lewis bases (acids) in order to reduce the interfacial surface energy, and vice versa ${ }^{17}$. We will assume that this coupling of Lewis acids and bases is linearly proportional to the magnitude of the electric field, so that the normalized polarization of dipoles becomes

$$
\alpha=\alpha(\lambda E)=L\left(\lambda \frac{\mu E}{k_{B} T}\right)
$$

where $\lambda$ is the induction coefficient of the Lewis acid-base coupling induced by the electric field. As shown in Fig. 2, the dipoles demonstrate a higher-order polarization through the induction effect $(\lambda=3.5)$; in addition, the orientation of dipoles saturates at high electric field.

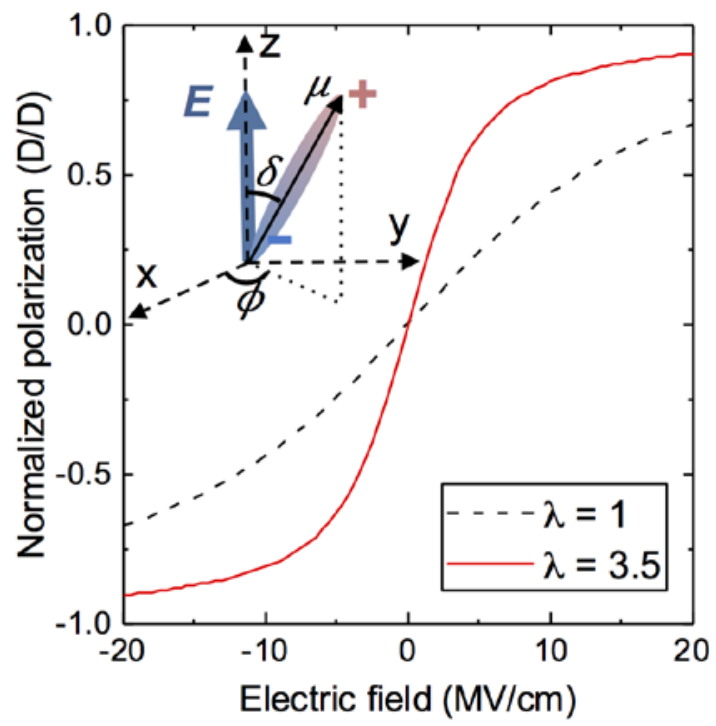

FIG. 2. The normalized average polarization of water dipoles with electric field without $(\lambda=1)$ and with $(\lambda=3.5)$ the induction effect. Inset: schematic diagram of the angular relationship between the dipole and the electric field in a spherical coordinate system.

The relationship between polar surface energy and electric field depends on the polarization of interfacial dipoles. We first find the liquid acid component of the surface energy; here only liquid dipoles with Lewis acids at the interface can 
contribute, where the rotational angle $\delta$ is from 0 to $\pi / 2$. In order to normalize the acid component at the PZC, i.e. $\gamma_{L}^{+}(0)=$ $\gamma_{L}^{+}$, this expression is divided by the fraction of corresponding dipoles at the PZC, i.e. $\int_{0}^{2 \pi} \int_{\pi / 2}^{0} \gamma_{L}^{+} n(\delta ; E) \sin \delta d \delta d \phi /$ $\int_{0}^{2 \pi} \int_{\pi / 2}^{0} n(\delta ; E) \sin \delta d \delta d \phi$. Similarly, we can obtain all the polar surface energy components under the influence of an electric field $E$, i.e.

$$
\begin{aligned}
& \gamma_{L}^{+}(\lambda E)=\gamma_{L}^{+} \frac{2}{1+\exp \left(-\lambda \frac{\mu_{L} E}{k_{B} T}\right)} \\
& \gamma_{L}^{-}(\lambda E)=\gamma_{L}^{-} \frac{2}{1+\exp \left(\lambda \frac{\mu_{L} E}{k_{B} T}\right)} \\
& \gamma_{S}^{+}(\lambda E)=\gamma_{S}^{+} \frac{2}{1+\exp \left(\lambda \frac{\mu_{S} E}{k_{B} T}\right)} \\
& \gamma_{S}^{-}(\lambda E)=\gamma_{S}^{-} \frac{2}{1+\exp \left(-\lambda \frac{\mu_{S} E}{k_{B} T}\right)}
\end{aligned}
$$

where $\mu_{\mathrm{L}}$ and $\mu_{\mathrm{S}}$ are the moments of liquid and solid dipoles, respectively. In Eqs. (4), the electric field is an external field, rather than a net field which accounts for the countering effect of polarized dipoles and is modified by a dielectric constant. This external field can be written as a multiplication of the net field in a layer and the corresponding dielectric constant, assuming that there is no trapped charge in the dielectric layers. In our case, according to Gauss's Law, we have $E=$ $\varepsilon_{r, S i N_{x}} E_{n e t, S i N_{x}}=\varepsilon_{r, C T Y O P} E_{n e t, C Y T O P}=\varepsilon_{r, \text { liquid }} E_{n e t, \text { liquid }}$, where $\varepsilon_{r, \text { SiN }}, \varepsilon_{r, \text { CYTOP }}$, and $\varepsilon_{r, \text { liquid }}$ are dielectric constants of $\mathrm{SiN}_{\mathrm{x}}, \mathrm{CYTOP}$, liquid, respectively, and $E_{\text {net,SiN }}, E_{\text {net,CYTOP }}, E_{\text {net,liquid }}$ are the net electric fields seen in SiN ${ }_{\mathrm{x}}$ CYTOP, liquid, respectively. Although the net fields seen at the solid interface and the liquid interface are different, the external fields at these interfaces are the same. In practice, the external electric field is the result of an externally applied voltage, so the electric field in Eqs. (4) needs to be substituted by the applied voltage. Due to high insulating quality of the CYTOP/SiN ${ }_{\mathrm{x}}$ dielectric layer and conducting property of the liquid, the voltage drop across the liquid can be negligible and the applied voltage can be written as $V=E_{n e t, C Y T O P} t_{C Y T O P}+E_{n e t, S i N_{x}} t_{\operatorname{SiN}_{x}}=E\left(t_{C T Y O P} / \varepsilon_{r, C T Y O P}+t_{\operatorname{SiN}_{x}} / \varepsilon_{r, S i N_{x}}\right)=E t / \varepsilon_{r}$, where $t_{\text {CTYOP }}$ and $t_{\text {SiN }_{x}}$ are the thicknesses of CYTOP and $\operatorname{SiN}_{\mathrm{x}}$, respectively, $t$ and $\varepsilon_{r}$ are the total thickness and the equivalent dielectric constant of CYTOP/SiN ${ }_{\mathrm{x}}$ dielectric layer. Therefore, the electric field in Eqs. (4) can be given by $E=\varepsilon_{r} V / t=$ $c_{i} V / \varepsilon_{0}$, where $c_{i}$ is the capacitance per unit area of CYTOP/SiN $\mathrm{N}_{\mathrm{x}}$ dielectric layer, $V$ is the applied voltage, $\varepsilon_{0}$ is the permittivity of free space.

The solid-liquid interfacial surface energy is determined by the properties of CYTOP, which can be found using the sessile drop technique with a range of probe liquids; here we use bipolar deionized (DI) water, monopolar ethylene glycol (EG), and nonpolar hexane, giving contacts angles of $110.4^{\circ}, 87.0^{\circ}$, and $15.5^{\circ}$, respectively. The surface energy components 
of these three liquids are listed in Table I. According the van Oss theory ${ }^{16}$, the surface energy components for CYTOP are shown in Table I.

TABLE I. Surface energy components of probe liquids and calculated data for CYTOP at the PZC (mJ m²).

\begin{tabular}{ccccc}
\hline \hline & $\gamma$ & $\gamma^{L W}$ & $\gamma^{+}$ & $\gamma^{-}$ \\
\hline water $^{18}$ & 72.8 & 21.8 & 25.5 & 25.5 \\
EG $^{18}$ & 48.0 & 29.0 & 3.0 & 30.1 \\
hexane $^{18}$ & 16.05 & 16.05 & 0 & 0 \\
CYTOP & 16.02 & 15.47 & 0.36 & 0.21 \\
\hline \hline
\end{tabular}

Figures 3(a) and (b) show the measured contact angle change of water and EG during electrowetting, along with theoretical curves for the classical model and the model developed here. The theoretical contact angle curves were calculated using the CYTOP parameter values determined earlier: measured capacitance per unit area $2.59 \times 10^{-4} \mathrm{~F} / \mathrm{m}^{2}$, and the dipole moment of water $1.85 \mathrm{D}$ and EG $2.01 \mathrm{D}^{19}$, respectively. Since CYTOP is rich in C-F bonds, the dipole moment was taken as the value for the $\mathrm{C}-\mathrm{F}$ bond, $1.41 \mathrm{D}^{20}$. The induction coefficient is an empirical value, $\lambda=3.5$. Both liquids show a high field saturation effect in the measurements, which is symmetric for water and asymmetric for EG due to their different polarities. Water is bipolar so that the acid component of surface energy is equal to the base component, but EG is monopolar, with the acid component of surface energy being smaller than the base component. With a positive voltage, the Lewis acids of EG are dominant at the interface, resulting in a higher interfacial surface energy and larger contact angle than for negative voltages. 

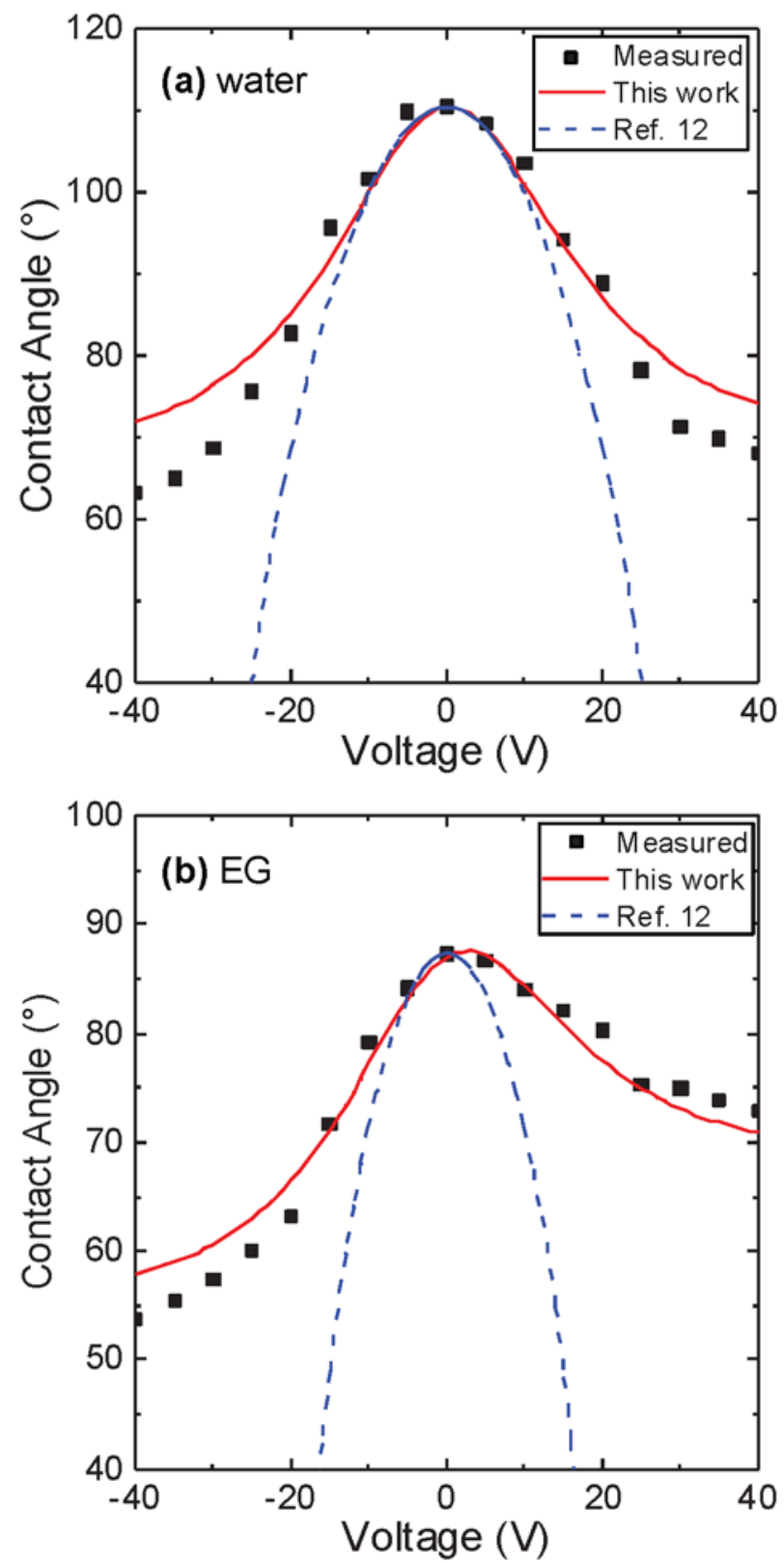

FIG. 3. Contact angle vs applied voltage during electrowetting for (a) water and (b) EG: measurements (squares), theoretical curves for this model (red solid) and the classical model (blue dash).

The high field saturation effect results from the saturation of the acid and the base surface energy components to zero at high field. Similar approaches were proposed by previous work, using non-negative contact angle ${ }^{21}$ and non-negative interfacial surface energy ${ }^{7,8,22}$ to define threshold voltage for contact angle saturation in dielectrophoresis and electrowetting, 
respectively. For dielectrophoresis, with consideration of electrical potential depth ${ }^{23}$ and dynamics of spreading ${ }^{21}$, the contact angle change and the rate of spreading were well modelled. However, in the case of electrowetting, where the contact angle change depends on the low dielectric constant of insulating dielectric layer rather than the high dielectric constant of liquid $^{21,23}$, the contact angle saturation is more severe. For electrowetting, although the contact angle saturation values and threshold voltage can be predicted by using non-negative interfacial surface energy, the change in contact angle in the transitional region remains unknown ${ }^{22,24}$. Here, our model based on dipole-dipole polarization describes the change of dipole orientation and interfacial surface energy over the whole field range, and the contact angle change is therefore predicted with good agreement over this field range.

In conclusion, a model for electrowetting based on dipole-dipole interactions at the solid-liquid interface has been developed and compared with the classical model for two situations. This model shows much better agreement with experimental results than the classical model at high voltage, as well as reproducing the observed asymmetry. The better fit to the experimental results is attributed to that the influence of polarization on the surface energy is considered, in particular the acid and the base surface energy components. When dipoles are polarized, the Lewis acids and the Lewis bases are ready to bind together to lower surface energy and therefore decrease the contact angle. In addition, the normalized polarization of dipole (or dipole orientation) will saturate at high field, leading to the saturation of the acid and the base surface energy components and hence the contact angle. The induction effect on the polarization of dipoles was assumed to be fixed; further improvement could be realized by accounting for the voltage dependence of this parameter.

${ }^{1}$ F. Mugele and J.-C. Baret, J. Phys. Condens. Matter 17, R705 (2005).

${ }^{2}$ S. Kuiper and B.H.W. Hendriks, Appl. Phys. Lett. 85, 1128 (2004).

${ }^{3}$ R.A. Hayes and B.J. Feenstra, Nature 425, 383 (2003).

${ }^{4}$ M.G. Pollack, R.B. Fair, and A.D. Shenderov, Appl. Phys. Lett. 77, 1725 (2000).

${ }^{5}$ R. Fobel, C. Fobel, and A.R. Wheeler, Appl. Phys. Lett. 102, 193513 (2013).

${ }^{6}$ H.J.J. Verheijen and M.W.J. Prins, Langmuir 15, 6616 (1999).

${ }^{7}$ V. Peykov, A. Quinn, and J. Ralston, Colloid Polym. Sci. 278, 789 (2000).

${ }^{8}$ A. Quinn, R. Sedev, and J. Ralston, J. Phys. Chem. B 109, 6268 (2005).

${ }^{9}$ M. Vallet, B. Berge, and L. Vovelle, Polymer. 37, 2465 (1996).

${ }^{10}$ D. Klarman, D. Andelman, R. Aviv, T. Aviv, and M. Urbakh, Langmuir 27, 6031 (2011).

${ }^{11}$ G. Lippmann, Ann. Chim. Phys. 5, 494 (1875). 
${ }^{12}$ B. Berge, C. R. Acad. Sci. Ser. II 317, 157 (1993).

${ }^{13}$ T. Young, Philos. Trans. R. Soc. London 95, 65 (1805).

${ }^{14}$ F.M. Fowkes, J. Phys. Chem. 66, 382 (1962).

${ }^{15}$ R.J. Good and L. a. Girifalco, J. Phys. Chem. 64, 561 (1960).

${ }^{16}$ C.J. Van Oss, M.K. Chaudhury, and R.J. Good, Chem. Rev. 88, 927 (1988).

${ }^{17}$ L.H. Lee, J. Adhes. Sci. Technol. 7, 583 (1993).

${ }^{18}$ C.J. Van Oss, Interfacial Forces in Aqueous Media, 2nd ed. (Taylor \& Francis, New York, 2006).

${ }^{19}$ W.M. Haynes, editor , CRC Handbook of Chemistry and Physics, 95th ed. (CRC Press, Boca Raton, 2014).

${ }^{20}$ P. Kirsch, Modern Fluoroorganic Chemistry: Synthesis, Reactivity, Applications (Wiley-VCH, Weinheim, 2004).

${ }^{21}$ G. McHale, C. V Brown, and N. Sampara, Nat. Commun. 4, 1605 (2013).

${ }^{22}$ M. Paneru, C. Priest, R. Sedev, and J. Ralston, J. Am. Chem. Soc. 132, 8301 (2010).

${ }^{23}$ G. McHale, C. V. Brown, M.I. Newton, G.G. Wells, and N. Sampara, Phys. Rev. Lett. 107, 186101 (2011).

${ }^{24}$ S. Chevalliot, S. Kuiper, and J. Heikenfeld, J. Adhes. Sci. Technol. 26, 1909 (2012). 